\title{
Multiattribute Choice With Ordinal Information: A Comparison of Different Decision Rules
}

\author{
Paula Sarabando and Luís Cândido Dias
}

\begin{abstract}
In the context of additive multiattribute aggregation, we address problems with ordinal information, i.e., considering a ranking of the weights (the scaling coefficients). Several rules for ranking alternatives in these situations have been proposed and compared, such as the rank-order-centroid weight, minimum value, central value, and maximum regret rules. This paper compares these rules, together with two rules that had never been studied (quasi-dominance and quasi-optimality) that use a tolerance parameter to extend the concepts of dominance and optimality. Another contribution of this paper is the study of the behavior of these rules in the context of selecting a subset of the most promising alternatives. This study intends to provide guidelines about which rules to choose and how to use them (e.g., how many alternatives to retain and what tolerance to use), considering the contradictory goals of keeping a low number of alternatives yet not excluding the best one. The comparisons are grounded on Monte Carlo simulations.
\end{abstract}

Index Terms-Imprecise/incomplete/partial information, Multiattribute utility theory (MAUT)/multiattribute value theory (MAVT), multicriteria decision analysis, ordinal information, simulation.

\section{INTRODUCTION}

$\mathbf{T}$ HIS WORK concerns the multicriteria selection problem, i.e., the problem of choosing one alternative from a list (a finite set) of alternatives that have been evaluated based on multiple and independent criteria. A large number of models/ methods have been proposed to support a decision maker (DM) dealing with such a problem, including the Multiattribute Value Theory (MAVT)/Multiattribute Utility Theory (MAUT), analytic hierarchy process, and outranking methods. (Recent comprehensive reviews are presented in [1] and [2].) Among these, we focus on the additive aggregation model in the context of MAUT/MAVT. In this model, the performance of an alternative on a given evaluation axis (which is usually called an attribute or a criterion) is measured by a value (or utility) function. The values (or utilities) attained by each alternative at the multiple functions are then summed and weighed by the scaling coefficients (which are usually known as weights)

Manuscript received January 3, 2008; revised May 26, 2008. First published February 24, 2009; current version published April 17, 2009. This work was supported by the Portuguese Foundation for Science and Technology (FCT/FEDER) under Grant POCI/EGE/58371/2004. This paper was recommended by Associate Editor E. Bass.

P. Sarabando is with the Escola Superior de Tecnologia de Viseu, 3504-510 Viseu, Portugal (e-mail: psarabando@mat.estv.ipv.pt).

L. C. Dias is with the INESC Coimbra R\&D Institute and the Faculty of Economics, University of Coimbra, 3004-512 Coimbra, Portugal (e-mail: 1dias@inescc.pt).

Color versions of one or more of the figures in this paper are available online at http://ieeexplore.ieee.org.

Digital Object Identifier 10.1109/TSMCA.2009.2014555 attached to those functions. This is one of the most well-known methods among practitioners and researchers, as it is simple to understand and its theoretical properties are well studied (e.g., see [1]-[6]). The same type of aggregation is used in other contexts, such as the computation of expected values, given the probability values (the seminal work of Fishburn [7] on imprecise information considers this context), and the aggregation of probabilities from experts using a linear opinion pool (e.g., [8]). Note, however, that many scholars do not endorse this type of additive aggregation, favoring alternatives, such as Surrogate Worth Tradeoffs or the use of reference points. (For a review of these approaches, see, e.g., [9].)

Building the value or utility functions for each criterion and setting the value of the weights require eliciting the preferences of a DM, which is often problematic. For him or her, value judgments are naturally easier to express through words than through numbers. Furthermore, preferences may evolve, as they are often unstable outcomes of unresolved internal conflicts in the DM's mind. Parameters such as the weights are artifacts whose semantic may be difficult to understand for the DM (which is related to the critical mistake of confusing these weights with importance; see [3]), and it is known that the way questions are posed (e.g., the questioning technique and the use of hierarchies) has a behavioral effect on the weights that are elicited [10]-[12]. In addition to these difficulties, other constraints of a more pragmatic nature may be present, e.g., the DM is reluctant in divulging precise parameter values about his preferences in public, his time and patience is rather limited, or the $\mathrm{DM}$ is, in fact, a group or an organization's representative who is unable to commit to precise values due to lack of consensus.

A related concern, according to Edwards et al. [13], [14], is that trying to elicit precise numerical values for the DM's preferences yields less reliable answers than trying to base the dialog on easier elicitation methods requiring less precise information. Furthermore, these authors argue that the decisionaiding process will be easier to understand and to be accepted by the DM, and also confirm that, due to a "flat-maxima" principle [5], the outcome of the analysis will not be much inferior to that of an analysis based on a rigorous elicitation of precise numerical values.

With the motivation brought by these considerations, a stream of research has been developed on the topic of decision making with imprecise (partial or incomplete) information, when the requirement for precise values of the parameters is replaced by constraints defining a set of parameter values that was deemed admissible. Similar to this paper, previous papers on this topic consider that only the weights are imprecise (e.g., [15]-[19]), address imprecise performance values 
(e.g., [20]), or are able to simultaneously deal with imprecise weights and performance values (e.g., [21]-[23]). Some of previous research in this context addresses group decisions (e.g., [24]-[27]). Dealing with imprecise information is also a subject of investigation in sorting problems (e.g., [28] and [29]).

Many approaches in dealing with imprecise information focus on the concepts of dominance and potential optimality. Dominance refers to a binary relation among alternatives: An alternative dominates another alternative if it is equally good or better for all the admissible values of the parameters and strictly better for at least one instance of such values. (Weaker definitions of dominance can also be used [30].) A nondominated alternative is potentially optimal if there exists an instance of admissible parameter values that makes it the best alternative. The idea behind these approaches (for a review, see, e.g., [21]) is to either screen the alternatives (keeping only the nondominated or potentially optimal ones) or derive a partial order on the set of alternatives based on the dominance relation. Among recent research trends, Malakooti [30] focused on the concept of preference strength and proposed an efficient simplex-based algorithm to screen or partially rank the alternatives for several types of linear constraints on global value. Ahn [31] extended this approach to allow continued use of the simplex-based algorithm when constraints are simultaneously placed on the weights and values for the additive model and to propose an algorithm for deriving a weak order based on the aggregation of the net flows of the value differences. Later, Ahn [32] addressed the problem of inferring weights, including the inference of the DMs' weights in group settings. Park [21] focused on computational efficiency, proposing mixed-integer formulations and the concepts of weak and strong potential optimality when weights and performances are imprecise. White and Holloway [23] considered a setting of progressive enrichment of information and proposed how to select the best question-response policy, after determining if there exists such a policy capable of identifying a single winner.

A different type of approach proposes decision rules to derive a complete ranking of the alternatives in the absence of precise information, such as the maximin rule or the rankorder-centroid (ROC) weight rule. (These and other rules are presented in Section II.) This stream of research has focused on assessing how good the proposed rules are, compared with an ideal situation in which all the parameters have known precise values. Usually, this is studied using Monte Carlo simulations: A large number of random problems (performances and weights) is generated, and the results obtained using these "true" values are compared with the results obtained by a rule based on only part of the information. The seminal work of Barons and Barrett [33] compared four rules based on surrogate weights to see how often the best alternative according to the rule coincided with the best alternative according to the "true" values (hit ratio) and the average loss of value when these did not coincide. Salo and Hämäläinen [18] used similar simulation experiments and a study of the probability of dominance to assess the effectiveness of eliciting imprecise information on weights and performances in the form of ratios of preference differences. The same type of experiments is used by Salo and Punkka [34] to compare novel types of constraints. Ahn and
Park [15] proposed two new rules and compared them with eight other rules in terms of both hit ratio and rank correlations, assuming ordinal weights.

This paper also uses Monte Carlo simulations to compare decision rules, with two innovative features. On the one hand, besides considering the rules previously studied (ROC weights, central value, maximin, and maximum regret), it includes two rules used by the Variable Interdependent Parameters Analysis (VIP Analysis) software [16] that have never been studied: 1) quasi-dominance and 2) quasi-optimality. On the other hand, contrarily to most previous research, we do not focus on a single best alternative according to the choice rule. Rather, we study the role of each rule in the context of a progressive reduction of alternatives (as suggested in [16] and [23]). Thus, our objective is to test how well the different rules behave if they are used to select a subset of alternatives (rather than a single one), trying to devise good (on average) strategies that conciliate the contradictory objectives of keeping a minimum number of alternatives and assuring that the chosen subset contains the best alternative. Our simulations cast some light on questions of which rule to use and how many alternatives should be kept (in the case of ROC weights, central value, maximin, and maximum regret) or what tolerance should be considered (in the case of quasi-dominance and quasi-optimality).

These experiments are designed to be comparable with previous studies. Hence, we test similar problem dimensions, and we consider, as done in many of the cited papers, that the imprecise information refers only to the weights of the additive model, which are usually considered to be the most difficult parameters to elicit. Furthermore, we restrict ourselves to the case where the elicited information about these parameters is ordinal, i.e., a rank order. This is the information required, for instance, by the SMARTER method [13], and it is often a first step when other methods (swings and tradeoffs) are used. Hence, we will also be able to relate our results to the theoretical analysis of Baucells et al. [35] for this type of cases with ordinal weights and known performances.

Section II introduces the mathematical notation and presents the tested rules. Section III describes the simulation plan, with the results being presented in Section IV. Section V concludes the paper, summarizing and discussing the main findings.

\section{Notation AND Decision Rule}

\section{A. Notation}

We will consider the evaluation of a discrete set of $m$ alternatives $A=\left\{a_{1}, \ldots, a_{m}\right\}$. The evaluation is first performed according to each criterion, considering a set of $n$ criteria (attributes) $X=\left\{x_{1}, \ldots, x_{n}\right\}$. Let $v_{i}($.$) denote the value func-$ tion (or utility function - the same would apply) corresponding to attribute $x_{i}$. Hence, $v_{i}\left(a_{j}\right) \in[0,1]$ denotes the value of alternative $a_{j}$ according to criterion $x_{i}$.

According to the additive aggregation model, the overall (multicriteria) value of an alternative $a_{j} \in A$ is given by

$$
v\left(a_{j}\right)=\sum_{i=1}^{n} w_{i} v_{i}\left(a_{j}\right)
$$


where $w_{i}$ is the weight (scaling coefficient) associated with $v_{i}$. These parameters are such that

$$
w_{1}, \ldots, w_{n} \geq 0 \quad \text { and } \quad \sum_{i=1}^{n} w_{i}=1 .
$$

As stated in the introduction, we are assuming that the single-criterion evaluations $v_{i}\left(a_{j}\right)$ are known $(i=1, \ldots, n$; $j=1, \ldots, m)$, whereas the only information about the weights is its rank order. Without loss of generality, we will consider that the criteria indexes are coded such that the weights are in decreasing order. Therefore, the set of weighing vectors compatible with this information is

$W^{*}=\left\{\left(w_{1}, w_{2}, \ldots w_{n}\right): w_{1} \geq w_{2} \geq \cdots \geq w_{n} \geq 0, \sum_{i=1}^{n} w_{i}=1\right\}$.

Given a set $W^{*}$ of weighing vectors, it is possible to compute three values.

1) Minimum value of an alternative $a_{j} \in A$ :

$$
v_{\min }\left(a_{j}\right)=\min _{w \in W^{*}} v\left(a_{j}\right) .
$$

2) Maximum value of an alternative $a_{j} \in A$ :

$$
v_{\max }\left(a_{j}\right)=\max _{w \in W^{*}} v\left(a_{j}\right) .
$$

3) Maximum difference of value of $a_{k} \in A$ over $a_{j} \in A$ :

$$
m_{k j}=\max _{w \in W^{*}}\left[v\left(a_{k}\right)-v\left(a_{j}\right)\right] .
$$

\section{B. Presentation of the Compared Decision Rules}

One of the possibilities described in the literature for dealing with the type of imprecise information we are addressing is to select a weighing vector from $W^{*}$ to represent that set and then use it to evaluate the alternatives. Examples of these are the use of equal weights, rank-sum weights, and ROC weights, which were compared in a simulation study by Barron and Barrett [33]. They concluded that the ROC weighing vector consistently provided better approximation than competing vectors. These weights are computed as

$$
w_{i}^{(\mathrm{ROC})}=\frac{1}{n} \sum_{j=i}^{n} \frac{1}{j}, \quad i=1, \ldots, n .
$$

Another type of rules that has been proposed is based on optimization, including three rules [18].

1) Maximin rule: This rule consists of evaluating each alternative by its minimum guaranteed (i.e., worst case) multicriteria value, choosing an alternative $a_{j}$ such that

$$
v_{\min }\left(a_{j}\right) \geq v_{\min }\left(a_{k}\right) \quad \forall a_{k} \in A .
$$

2) Maximum loss of value or minimax regret rule: This rule consists of evaluating each alternative by the maximum

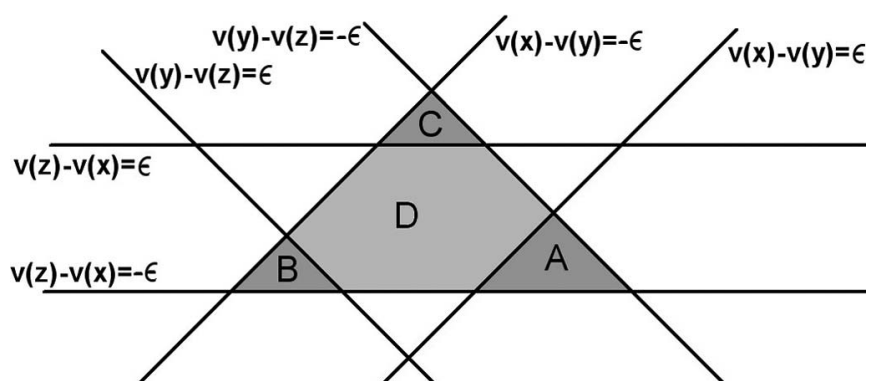

Fig. 1. Possibility of cycles in the quasi-dominance relation.

loss of value with respect to a better alternative (i.e., the worst-case regret), choosing an alternative $a_{j}$ such that

$$
\max _{a_{k} \neq a_{j}} m_{k j} \leq \max _{a_{k} \neq a_{l}} m_{k l} \quad \forall a_{l} \in A .
$$

3) Central value rule: This rule consists of evaluating each alternative by the midpoint of the interval of possible multicriteria value, choosing an alternative $a_{j}$ such that

$$
v_{\min }\left(a_{j}\right)+v_{\max }\left(a_{j}\right) \geq v_{\min }\left(a_{k}\right)+v_{\max }\left(a_{k}\right) \quad \forall a_{k} \in A .
$$

Finally, we have considered two new rules based on the concepts of quasi-optimality and quasi-dominance [16].

1) Quasi-optimality rule: This rule consists of choosing the subset of alternatives $A_{\epsilon}^{o}$ (which may be empty or may contain more than one alternative) that are better than or almost equal to all other alternatives when a tolerance $\epsilon>0$ is taken into account, i.e.,

$$
a_{j} \in A_{\epsilon}^{o} \Leftrightarrow m_{k j} \leq \epsilon \quad \forall a_{k} \in A .
$$

A comparison of (9) and (11) shows that, if the maximum regret associated with an alternative is $\epsilon$ or less, then it is quasi-optimal with tolerance $\epsilon$. If $\epsilon=0$, then condition (11) defines an optimal alternative.

2) Quasi-dominance rule: This rule consists of choosing the subset of alternatives $A_{\epsilon}^{n}$ that are not quasi-dominated by any other alternative. The definition of quasi-dominance with tolerance $\epsilon>0$ is given as follows:

$$
\begin{aligned}
& a_{i} \text { quasi-dominates } a_{j}\left(a_{i} \Delta_{\epsilon} a_{j}\right) \\
& \Leftrightarrow m_{j i}<0 \vee\left(m_{j i} \leq \epsilon \wedge m_{i j}>\epsilon\right) .
\end{aligned}
$$

Hence, according to this rule, the set of alternatives that should be selected is

$$
A_{\epsilon}^{n}=\left\{a_{j} \in A: \neg a_{i} \Delta_{\epsilon} a_{j}, \forall a_{i} \in A\right\} .
$$

Note that $A_{\epsilon}^{n}$ may often contain more than one alternative, but, in some rarer cases, it may be empty. This may occur when there exist cycles, as shown in Fig. 1 for a case with three alternatives $x, y$, and $z$. Also shown in the figure are several lines (linear equalities) in weight-space-defining regions $A, B, C$, and $D$. The region above line $v(z)-v(x)=\epsilon$, which includes the inner triangle $C$, represents the weighing vectors that lead to a victory $z$ over $x$ by a margin greater than $\epsilon$. The 
region below line $v(z)-v(x)=-\epsilon$ represents the weighing vectors that lead to a victory of $x$ over $z$ by a margin greater than $\epsilon$. Hence, if the set $W^{*}$ of acceptable weighing vectors intersects the region above line $v(z)-v(x)=\epsilon$ without intersecting the region below line $v(z)-v(x)=-\epsilon$, then there exist weighing vectors in $W^{*}$ that make $z$ clearly better than $x$, whereas the reverse is not true; hence, $z$ quasi-dominates $x$ for tolerance $\epsilon$. Following an analogous reasoning for pairs $(x, y)$ and $(y, z)$, one can conclude that, if $W^{*}$ is contained in the triangle $A \cup B \cup C \cup D$ and intersects each of the triangles $A$, $B$, and $C$, then we have a cycle in the quasi-dominance relation: $z \Delta_{\epsilon} x, x \Delta_{\epsilon} y$, and $y \Delta_{\epsilon} z$. If $\epsilon=0$, then condition (12) basically yields the (strict) dominance relation.

Other rules were also used in the simulations (the use of equal weights and maximax rule) but consistently provided worse results (see details in [36]); hence, they were omitted in this paper.

\section{Planning of the Simulation Experiments}

A sequence of experiments using Monte Carlo simulations was conceived to compare the different rules presented in the previous section in the context of selecting a promising subset of alternatives, given ordinal information about the weights of an additive model. We considered problems with 5,10 , and 15 criteria, and 5, 10, 15, 25, and 50 alternatives. (These dimensions are similar to those considered in [15] and [18].) Similarly to [18], we generated 5000 random problem instances for each problem dimension, checking that the use of a larger number of problem instances did not significantly affect the results.

Single-criterion values $v_{i}\left(a_{j}\right)$ were generated from a uniform distribution in the interval $[0,1]$ and then normalized criterionwise such that the highest value in each criterion would be 1 and the lowest value would be 0 . For each criterion, suppose that $v_{i}^{\text {lo }}$ and $v_{i}^{\text {hi }}$ were the lowest and highest values among the $m$ values generated, respectively. Then, the normalized value of $v_{i}\left(a_{j}\right)$ is equal to $\left(v_{i}\left(a_{j}\right)-v_{i}^{\mathrm{lo}}\right) /\left(v_{i}^{\mathrm{hi}}-v_{i}^{\mathrm{lo}}\right)$. The uniform distribution was also considered in [15] and [18]. Barron and Barrett [33] used both uniform and normal distributions to compare the hit ratios and loss of value of four rules, concluding that using the normal distribution did not significantly influence the results.

The weights were also generated according to a uniform distribution on $W^{*}$ using the process described in [37]. To generate the weights for the $n$-attribute case, we draw $n-1$ independent random numbers from a uniform distribution on $(0,1)$ and rank these numbers. Suppose that the ranked numbers are $r_{(n-1)} \geq \cdots \geq r_{(2)} \geq r_{(1)}$. The following differences can then be obtained: $w_{n}=1-r_{(n-1)}, w_{n-1}=$ $r_{(n-1)}-r_{(n-2)}, \ldots$, and $w_{1}=r_{(1)}-0$. Then, the set of numbers $\left(w_{1}, w_{2}, \ldots, w_{n}\right)$ will add up to 1 and will be uniformly distributed on the unit simplex defined by the rank-order constraints (3) after relabeling. Note that, as confirmed in [33], if the information set on the weights is specified entirely by a complete ranking of the alternatives, then no point in $W^{*}$ may be considered more likely than another, and the density of the weights is uniform over $W^{*}$.
For each random problem instance defined by a table of single-criterion values and a vector of weights, the additive model yields the multicriteria value of each alternative. From these global values, we derive what we may call the supposedly true ranking, i.e., the ranking one would obtain if all this cardinal information was used. On the other hand, the ROC weight, maximin, minimax regret, and central value rules produce rankings using only the ordinal information about the weights. Our first set of experiments compared these rankings, whereas the remaining experiments concerned quasi-dominance and quasi-optimality.

\section{A. First Set of Experiments}

According to the problem dimensions and the problem generation process previously described, we computed, for each generated problem, the supposedly true ranking and the rankings provided by the ROC weights, central value, minimax regret, and maximin rules. Comparing the supposedly true ranking and the rule's ranking, we sought to compute two results.

1) The rank that the best alternative of the supposedly true ranking attains in the ranking generated by the rule. This allows us to know the minimum number of alternatives that should be chosen, starting from the top of the rule's ranking to ensure that the supposedly best alternative is included in the chosen set. Such results generalize existing results [18], [33], [34] concerning the hit ratio, i.e., the proportion of times that the best alternatives in the two rankings coincide.

2) The rank that the best alternative of the ranking generated by the rule attains in the supposedly true ranking. This allows knowing how good the rule's chosen alternative is in terms of the supposedly true ranking. Such results complement existing results [18], [33], [34] concerning the "loss of value."

\section{B. Second Set of Experiments}

According to the problem dimensions and the problem generation process previously described, we computed, for each generated problem, the number of optimal alternatives (tolerance $\epsilon=0$ ) and the number of quasi-optimal alternatives for different values of tolerance parameter $\epsilon$. (We have considered tolerances of $0.03,0.05,0.1$, and 0.2.) Furthermore, to assess the quality of these alternatives with respect to the supposedly true ranking, we determined the position of the best ranked alternative kept if only the quasi-optimal alternatives are retained.

\section{Third Set of Experiments}

In experiments analogous to the second set, we computed, for each generated problem, the number of dominated (tolerance $\epsilon=0$ ) and quasi-dominated alternatives for the same values of $\epsilon$; furthermore, we determined the position of the best ranked alternative kept if all the quasi-dominated ones were excluded. 
TABLE I

Position of the Best Alternative According to the ROC Weight Rule, Maximin Rule, Minimax Regret Rule, and Central Weight Rule in the Supposedly True Ranking ( $n$ AND $m$ Denote the Number of Criteria and the Number of Alternatives, Respectively)

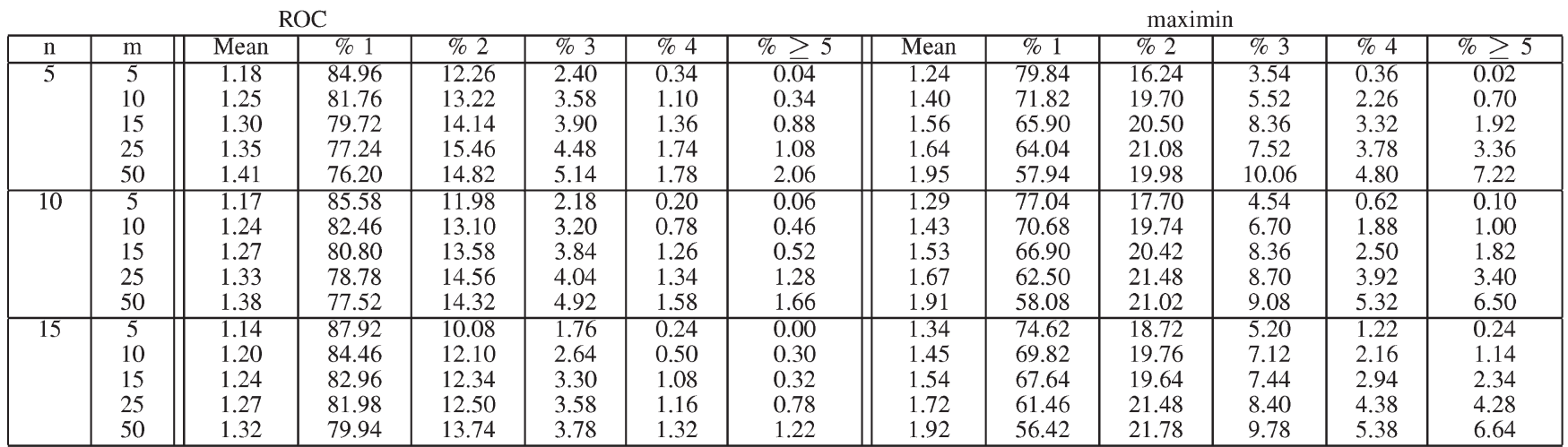

minimax regret

\begin{tabular}{|c|c|c|c|c|c|c|c|c|c|c|c|c|c|}
\hline & & & & & & & & & & & & & \\
\hline $\mathrm{n}$ & $\mathrm{m}$ & Mean & $\% 1$ & $\% 2$ & $\% 3$ & $\% 4$ & $\% \geq 5$ & Mean & $\% 1$ & $\% 2$ & $\% 3$ & $\% 4$ & $\% \geq 5$ \\
\hline \multirow[t]{5}{*}{5} & 5 & 1.26 & 79.66 & 15.88 & 3.82 & 0.56 & $0 . \overline{08}$ & 1.26 & 79.78 & 15.36 & 3.92 & 0.78 & 0.16 \\
\hline & 10 & 1.40 & 73.80 & 17.60 & 5.40 & 1.96 & 1.24 & 1.41 & 74.28 & 16.26 & 5.74 & 2.30 & 1.42 \\
\hline & 15 & 1.49 & 70.34 & 18.02 & 6.74 & 3.30 & 1.60 & 1.52 & 71.14 & 17.14 & 5.98 & 2.86 & 2.88 \\
\hline & 25 & 1.59 & 66.50 & 19.68 & 7.38 & 3.50 & 2.94 & 1.64 & 67.68 & 18.34 & 6.42 & 3.30 & 4.26 \\
\hline & 50 & 1.69 & 64.52 & 18.42 & 8.68 & 4.24 & 4.14 & 1.81 & 63.98 & 18.24 & 8.18 & 3.88 & 5.72 \\
\hline \multirow[t]{5}{*}{10} & 5 & 1.38 & 73.18 & 18.18 & 6.28 & 2.00 & 0.36 & 1.40 & 72.24 & 18.74 & 6.10 & 2.26 & 0.66 \\
\hline & 10 & 1.57 & 66.86 & 19.46 & 7.70 & 3.44 & 2.54 & 1.68 & 64.56 & 19.12 & 7.80 & 4.10 & 4.42 \\
\hline & 15 & 1.69 & 62.82 & 20.42 & 8.84 & 4.06 & 3.86 & 1.87 & 59.62 & 19.84 & 9.26 & 4.44 & 6.84 \\
\hline & 25 & 1.83 & 60.60 & 19.70 & 9.08 & 4.86 & 5.76 & 2.02 & 58.16 & 19.10 & 9.74 & 4.18 & 8.82 \\
\hline & 50 & 1.99 & 56.68 & 21.02 & 9.56 & 4.86 & 7.88 & 2.30 & 53.58 & 19.06 & 10.44 & 5.38 & 11.54 \\
\hline \multirow[t]{5}{*}{15} & 5 & 1.48 & 67.04 & 21.98 & 7.66 & 2.58 & 0.74 & 1.52 & 66.96 & 20.22 & 8.30 & 3.02 & 1.50 \\
\hline & 10 & 1.73 & 60.64 & 21.24 & 5.18 & 4.76 & 4.18 & 1.89 & 56.68 & 20.84 & 10.66 & 5.70 & 6.12 \\
\hline & 15 & 1.88 & 57.10 & 22.04 & 10.02 & 4.88 & 5.96 & 2.10 & 54.44 & 20.00 & 10.40 & 5.82 & 9.34 \\
\hline & 25 & 2.10 & 53.08 & 21.04 & 11.36 & 5.72 & 8.80 & 2.33 & 50.88 & 20.60 & 10.32 & 6.28 & 11.92 \\
\hline & 50 & 2.33 & 50.36 & 19.98 & 10.80 & 7.22 & 11.64 & 2.76 & 46.18 & 19.22 & 10.86 & 7.28 & 16.46 \\
\hline
\end{tabular}

\section{RESUlT OF THE EXPERIMENTS}

\section{A. Ranking Rules}

A set of experiments was performed to see how the different rules compare. To assess a strategy for selecting the best alternative according to each rule, we looked at the position attained by the alternative suggested by the rule in the supposedly true ranking. Detailed results concerning the rank that the best alternative, according to the ROC weight, maximin, minimax regret, and central weight rules, attains in the supposedly true ranking are presented in Table I, which indicates, for each rule and each problem dimension, the mean position in the supposedly true ranking (the minimum was always 1 ) and the proportion of cases where the position was 1, 2, 3, 4, or higher. Column $\% 1$ in Table I presents the hit ratio of the rules, i.e., the proportion of cases where the rule selects the supposedly best alternative, allowing us to draw some conclusions.

1) Results indicate that the ROC weight rule is the best rule for this strategy, with a mean hit ratio of 0.8149 , which is greater than the mean ratios for the maximin (0.6698), minimax regret $(0.6421)$, and central weight $(0.6268)$ rules. We used paired t-tests to see if a significant difference occurs in hit ratios produced between the ROC rule and other rules. Comparing the ROC and maximin rules, we obtained a t-statistic of 11.831 and a p-value of 0 , meaning that the ROC rule significantly outperforms the maximin rule for all significance levels. Comparing the ROC rule with the minimax regret and central weight rules, we obtained a t-statistic of 8.724 and 7.976, respectively, and p-values of 0 .
2) There is not much difference among the results for the maximin, minimax regret, and central weight rules, all of which offer reasonable results (but worse than the ROC weight rule); for problems with five criteria, the central weight rule was the best of the three, but it was the worst for problems with 10 or 15 criteria.

3) Using ROC weights, the hit ratio moderately decreased as the number of alternatives increased and slightly increased as the number of criteria increased. For the remaining rules, the hit ratio more steeply decreased as the number of alternatives increased and decreased as the number of criteria increased.

Table I is also useful in assessing how the different rules compare when, rather than considering the probability of getting the supposedly best alternative (which is given by the hit ratio), we consider the probability of getting one of the top-ranked alternatives of the true ranking. For instance, the alternative suggested by the ROC rule is one of the two best alternatives in at least $90 \%$ of the cases, whereas this probability would noticeably be lower for the remaining rules (cf. the sum of columns $\% 1$ and $\% 2$ ).

In a strategy for progressive reduction of the number of alternatives, one would wish to retain as few alternatives as possible for further analysis and yet not discard the best one. To know how many alternatives should be kept, we need to know the position of the supposedly best alternative in the ranking induced by each rule. Table II indicates, for each problem dimension, the mean position of the supposedly best alternative in each rule's ranking (the minimum was always 1) and the proportion of cases where the position was $1,2,3,4$, or 
TABLE II

Position of the Supposedly Best Alternative in the RANKing Induced By the ROC Weight Rule, Maximin Rule, Minimax Regret Rule, and Central Weight Rule

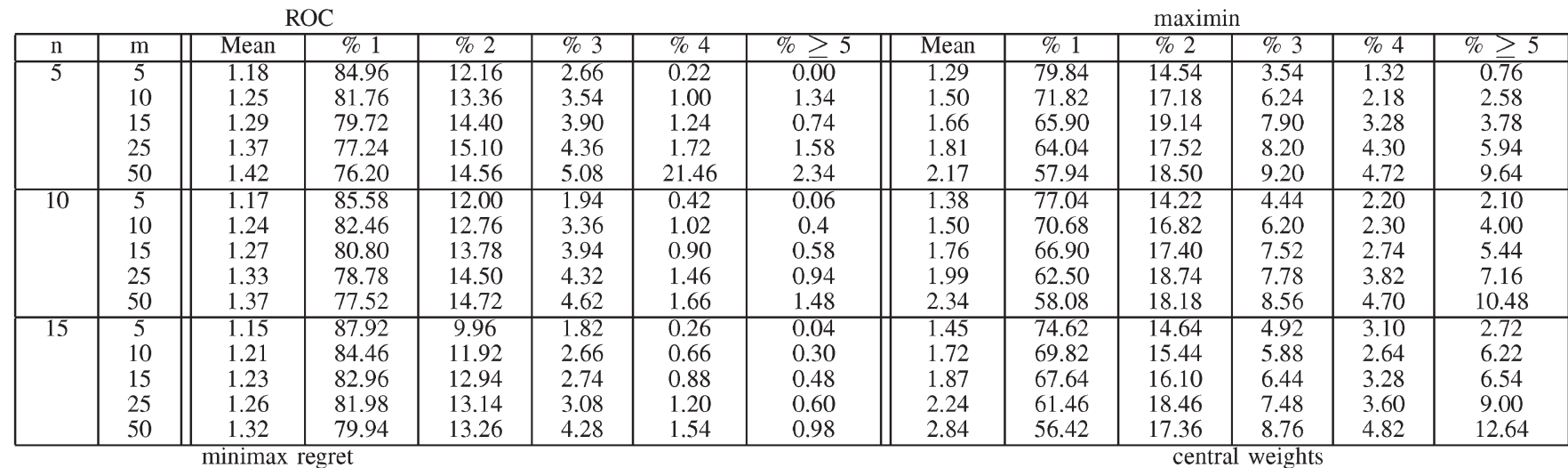

\begin{tabular}{|c|c|c|c|c|c|c|c|c|c|c|c|c|c|}
\hline & & & & & & & & & & & & & \\
\hline $\mathrm{n}$ & $\mathrm{m}$ & Mean & $\% 1$ & $\% 2$ & $\% 3$ & $\% 4$ & $\% \geq 5$ & Mean & $\% 1$ & $\% 2$ & $\% 3$ & $\% 4$ & $\% \geq 5$ \\
\hline \multirow[t]{5}{*}{5} & 5 & 1.32 & 79.66 & 12.46 & 4.85 & 2.26 & $0 . \overline{76}$ & 1.27 & 79.78 & 15.06 & 4.00 & 0.90 & $0 . \overline{26}$ \\
\hline & 10 & 1.56 & 73.80 & 12.98 & 5.94 & 2.88 & 4.40 & 1.39 & 74.28 & 17.34 & 5.34 & 1.76 & 1.28 \\
\hline & 15 & 1.72 & 70.34 & 14.04 & 6.06 & 3.48 & 6.08 & 1.50 & 71.14 & 17.24 & 7.08 & 2.08 & 2.46 \\
\hline & 25 & 1.89 & 66.50 & 16.26 & 6.76 & 3.46 & 7.02 & 1.62 & 67.68 & 19.06 & 7.02 & 2.60 & 3.64 \\
\hline & 50 & 2.16 & 64.52 & 15.60 & 6.58 & 3.82 & 9.48 & 1.81 & 63.98 & 18.06 & 8.38 & 3.68 & 5.90 \\
\hline \multirow[t]{5}{*}{10} & 5 & 1.48 & 73.18 & 13.46 & 7.16 & 4.10 & 2.10 & 1.40 & 72.24 & 19.04 & 6.30 & 1.78 & 0.67 \\
\hline & 10 & 1.84 & 66.86 & 13.94 & 6.82 & 4.22 & 8.16 & 1.65 & 64.56 & 20.92 & 7.42 & 3.40 & 3.70 \\
\hline & 15 & 2.19 & 62.82 & 14.06 & 6.10 & 4.56 & 11.46 & 1.85 & 59.62 & 20.90 & 9.10 & 4.58 & 10.00 \\
\hline & 25 & 2.57 & 60.6 & 13.74 & 7.02 & 3.88 & 14.76 & 2.06 & 58.16 & 18.88 & 9.12 & 5.26 & 8.58 \\
\hline & 50 & 3.29 & 56.68 & 14.02 & 7.34 & 4.22 & 17.74 & 2.44 & 53.58 & 19.78 & 9.80 & 4.94 & 11.90 \\
\hline \multirow[t]{5}{*}{15} & 5 & 1.60 & 67.04 & 16.04 & 9.08 & 5.12 & 2.72 & 1.50 & 66.96 & 21.04 & 8.10 & 3.04 & 0.86 \\
\hline & 10 & 2.12 & 60.64 & 14.14 & 7.90 & 5.54 & 11.78 & 1.86 & 56.68 & 22.94 & 9.72 & 4.86 & 5.80 \\
\hline & 15 & 2.52 & 57.10 & 13.92 & 7.96 & 5.14 & 15.88 & 2.10 & 54.44 & 21.14 & 9.96 & 5.42 & 9.30 \\
\hline & 25 & 3.24 & 53.08 & 13.62 & 7.44 & 5.26 & 20.60 & 2.53 & 50.88 & 18.88 & 9.88 & 6.26 & 14.10 \\
\hline & 50 & 4.39 & 50.36 & 13.02 & 7.54 & 4.94 & 24.14 & 3.05 & 46.18 & 18.06 & 10.50 & 7.14 & 18.12 \\
\hline
\end{tabular}
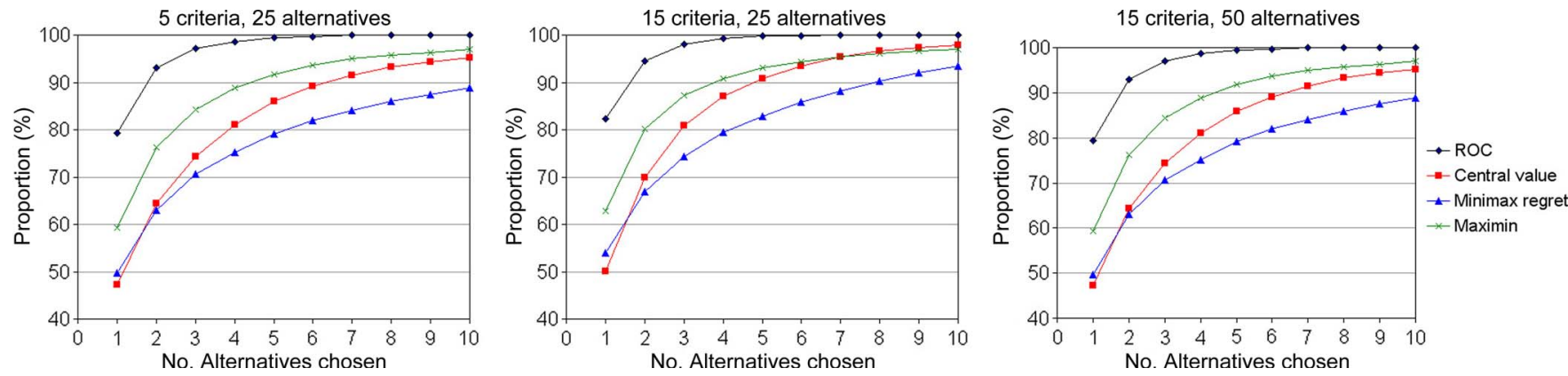

Fig. 2. Proportion of cases (in percent) where the best alternative is chosen as the number of retained alternatives increases.

higher. Fig. 2, which clearly shows the superiority of the ROC rule, shows how the probability of keeping the supposedly best alternative increases with the number of alternatives that were retained.

- Using the ROC rule, for these problem dimensions, selecting two alternatives would suffice in $90 \%$ of the cases. Selecting three alternatives would not suffice only in 5\% of the cases. For the other rules, we have to select more alternatives, especially as the problem dimension increases.

\section{B. Quasi-Optimality Rule}

The second set of experiments was performed to study a strategy for selecting a subset of alternatives. However, rather than ranking the alternatives according to some rule and retaining a prespecified number of top-ranked alternatives, as in Section IV-A, we will consider retaining an unknown number of alternatives satisfying a given condition.
The first condition that we tested was quasi-optimality for a given tolerance $\epsilon$ (recall from Section II-B that a quasi-optimal alternative is an alternative that cannot be defeated by another one by a difference greater than the allowed tolerance). Our purpose was to observe whether there is a good value for the tolerance such that it would allow to retain few alternatives without discarding the supposedly best one. In these experiments, we sought to know how many alternatives would be retained, as well as the best position (in terms of the supposedly true ranking) reached by the retained alternatives. Table III indicates the mean number of optimal and quasi-optimal alternatives for different tolerances $\epsilon$ and the proportion of cases with 0,1 , or more quasi-optimal alternatives. Conclusions are summarized here.

1) There are many cases, particularly for low tolerance values, with no quasi-optimal alternatives (Fig. 3). However, when there exist quasi-optimal alternatives, they are rather few, and there is a very high probability 
TABLE III

Number of Optimal (Tolerance $\epsilon=0$ ) OR Quasi-Optimal Alternatives (Tolerance $\epsilon=0.05, \epsilon=0.1$, AND $\epsilon=0.2$ )

\begin{tabular}{|c|c|c|c|c|c|c|c|c|c|c|c|c|c|c|c|c|}
\hline \multicolumn{4}{|c|}{$\epsilon=0$} & \multicolumn{4}{|c|}{$\epsilon=0.05$} & \multicolumn{5}{|c|}{$\epsilon=0.1$} & \multicolumn{4}{|c|}{$\epsilon=0.2$} \\
\hline $\mathrm{n}$ & $\mathrm{m}$ & Mean & $\% 0$ & $\% 1$ & Mean & $\% 0$ & $\% 1$ & $\% 2$ & Mean & $\% 0$ & $\% 1$ & $\% \geq 2$ & Mean & $\% 0$ & $\% 1$ & $\% \geq 2$ \\
\hline 5 & 5 & 0.20 & 79.96 & 20.04 & 0.30 & 70.38 & 29.62 & 0.00 & 0.42 & 58.30 & 41.56 & $0 . \overline{14}$ & 0.75 & 29.70 & 65.28 & $5 . \overline{02}$ \\
\hline & 10 & 0.11 & 89.24 & 10.76 & 0.22 & 78.24 & 21.74 & 0.02 & 0.40 & 61.20 & 38.10 & 0.70 & 0.99 & 21.92 & 59.48 & 18.60 \\
\hline & 15 & 0.07 & 92.78 & 7.22 & 0.18 & 81.76 & 18.18 & 0.06 & 0.39 & 62.64 & 35.98 & 1.38 & 1.20 & 16.70 & 52.92 & 30.38 \\
\hline & 25 & 0.04 & 96.08 & 3.92 & 0.17 & 83.54 & 16.38 & 0.08 & 0.42 & 60.66 & 36.88 & 2.46 & 1.59 & 10.78 & 40.20 & 49.02 \\
\hline & 50 & 0.02 & 97.86 & 2.14 & 0.14 & 85.84 & 14.08 & 0.08 & 0.49 & 55.72 & 39.92 & 4.36 & 2.35 & 4.06 & 23.10 & 72.84 \\
\hline 10 & 5 & 0.11 & 89.24 & 10.76 & 0.18 & 82.24 & 17.76 & 0.00 & 0.29 & 71.20 & 28.80 & 0.00 & 0.62 & 40.70 & 56.72 & 2.58 \\
\hline & 10 & 0.04 & 95.82 & 4.18 & 0.11 & 88.8 & 11.20 & 0.00 & 0.25 & 75.56 & 24.32 & 0.12 & 0.80 & 32.76 & 55.76 & 11.48 \\
\hline & 15 & 0.02 & 97.70 & 2.30 & 0.09 & 91.24 & 8.76 & 0.00 & 0.24 & 76.80 & 22.88 & 0.32 & 0.97 & 26.40 & 53.20 & 20.40 \\
\hline & 25 & 0.01 & 98.76 & 1.24 & 0.07 & 92.82 & 7.18 & 0.00 & 0.23 & 77.98 & 21.50 & 0.52 & 1.23 & 19.76 & 47.30 & 32.94 \\
\hline & 50 & 0.01 & 99.54 & 0.46 & 0.05 & 94.96 & 5.04 & 0.00 & 0.18 & 82.56 & 17.36 & 0.08 & 1.73 & 11.42 & 33.28 & 55.30 \\
\hline 15 & 5 & 0.07 & 93.00 & 7.00 & 0.13 & 86.74 & 13.26 & 0.00 & 0.25 & 75.44 & 24.56 & 0.00 & 0.58 & 44.90 & 52.72 & 2.36 \\
\hline & 10 & 0.03 & 97.40 & 2.60 & 0.08 & 92.08 & 7.92 & 0.00 & 0.20 & 80.36 & 19.60 & 0.04 & 0.75 & 36.20 & 53.60 & 2.38 \\
\hline & 15 & 0.01 & 98.88 & 1.12 & 0.06 & 94.42 & 5.58 & 0.00 & 0.18 & 82.14 & 17.72 & 0.14 & 0.89 & 30.80 & 51.48 & 10.20 \\
\hline & 25 & 0.01 & 99.34 & 0.66 & 0.04 & 95.66 & 4.34 & 0.00 & 0.17 & 83.16 & 16.58 & 0.26 & 1.13 & 23.60 & 47.02 & 29.38 \\
\hline & 50 & 0.00 & 99.92 & 0.08 & 0.03 & 96.94 & 3.06 & 0.00 & 0.17 & 83.36 & 16.20 & 0.44 & 1.59 & 13.44 & 38.52 & 48.04 \\
\hline
\end{tabular}
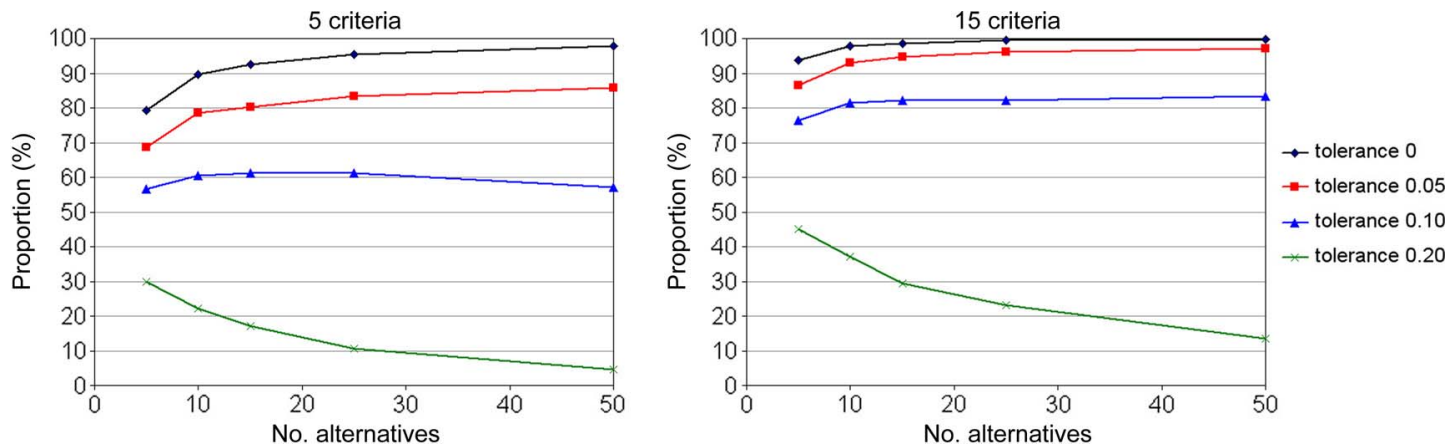

Fig. 3. Proportion of cases (in percent) with no quasi-optimal alternatives. (Left) Five criteria. (Right) 15 criteria.
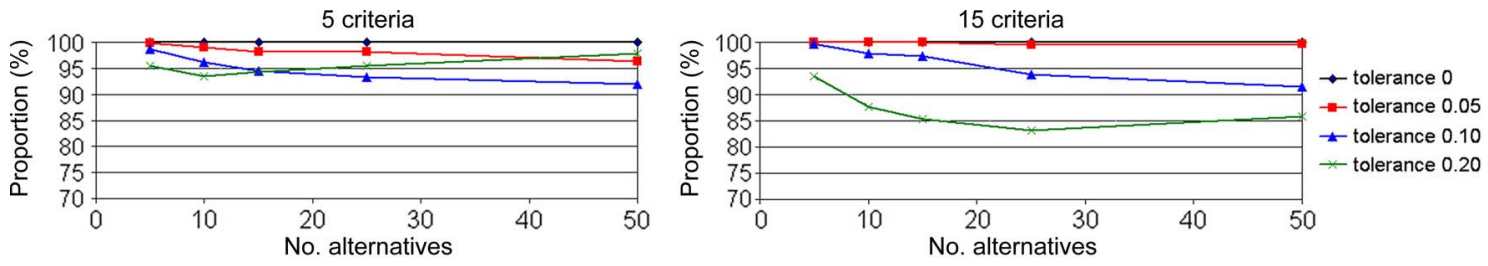

Fig. 4. Proportion of cases (in percent) where the best alternative is quasi-optimal (cases with 5 and 15 criteria).

TABLE IV

Best Position Attained By the Quasi-Optimal Alternatives (Tolerance $\epsilon=0.05, \epsilon=0.1$, And $\epsilon=0.2$ )

\begin{tabular}{|c|c|c|c|c|c|c|c|c|c|c|c|c|c|}
\hline \multicolumn{4}{|c|}{$\epsilon=0.05$} & \multirow{2}{*}{\multicolumn{5}{|c|}{$\epsilon=0.1$}} & \multicolumn{5}{|c|}{$\epsilon=0.2$} \\
\hline$n$ & $\mathrm{~m}$ & Mean & $\% 1$ & & & Mean & $\% 1$ & & $\% \geq 3$ & Mean & $\% 1$ & $\% 2$ & $\% \geq 3$ \\
\hline 5 & 5 & 1.00 & 99.75 & 0.25 & 0.00 & 1.01 & 98.62 & 1.29 & $0 . \overline{09}$ & 1.05 & 95.40 & 4.21 & $0 . \overline{39}$ \\
\hline & 10 & 1.01 & 99.06 & 0.90 & 0.04 & 1.04 & 96.13 & 3.68 & 0.19 & 1.08 & 93.48 & 5.65 & 0.87 \\
\hline & 15 & 1.02 & 98.10 & 1.86 & 0.04 & 1.06 & 94.52 & 5.19 & 0.29 & 1.07 & 94.22 & 4.65 & 1.13 \\
\hline & 25 & 1.02 & 98.17 & 1.72 & 0.11 & 1.08 & 93.31 & 5.70 & 0.99 & 1.06 & 95.47 & 3.74 & 0.79 \\
\hline & 50 & 1.04 & 96.24 & 3.62 & 0.14 & 1.10 & 91.96 & 6.39 & 1.65 & 1.03 & 97.84 & 1.84 & 0.32 \\
\hline 10 & 5 & 1.00 & 100.00 & 0.00 & 0.00 & 1.01 & 99.21 & 0.79 & 0.00 & 1.06 & 94.86 & 4.78 & 0.36 \\
\hline & 10 & 1.00 & 99.89 & 0.11 & 0.00 & 1.03 & 97.56 & 2.33 & 0.11 & 1.11 & 90.82 & 7.69 & 1.39 \\
\hline & 15 & 1.00 & 99.66 & 0.34 & 0.00 & 1.04 & 96.16 & 3.63 & 0.21 & 1.14 & 89.08 & 8.71 & 2.24 \\
\hline & 25 & 1.00 & 99.56 & 0.44 & 0.00 & 1.06 & 94.67 & 4.90 & 0.43 & 1.15 & 88.93 & 8.41 & 1.93 \\
\hline & 50 & 1.01 & 99.00 & 1.00 & 0.00 & 1.10 & 90.76 & 8.28 & 0.96 & 1.10 & 92.18 & 6.12 & 2.66 \\
\hline 15 & 5 & 1.00 & 100.00 & 0.00 & 0.00 & 1.00 & 99.62 & 0.38 & 0.00 & 1.07 & 93.47 & 6.28 & 0.25 \\
\hline & 10 & 1.00 & 100.00 & 0.00 & 0.00 & 1.02 & 97.80 & 2.20 & 0.00 & 1.15 & 87.71 & 10.06 & 2.20 \\
\hline & 15 & 1.00 & 100.00 & 0.00 & 0.00 & 1.03 & 97.40 & 2.56 & 0.04 & 1.19 & 85.38 & 11.40 & 3.52 \\
\hline & 25 & 1.00 & 99.52 & 0.48 & 0.00 & 1.07 & 93.82 & 5.67 & 0.51 & 1.25 & 83.09 & 11.98 & 4.93 \\
\hline & 50 & 1.00 & 99.61 & 0.39 & 0.00 & 1.10 & 91.52 & 7.52 & 0.96 & 1.21 & 85.81 & 10.29 & 3.90 \\
\hline
\end{tabular}

that the supposedly best alternative is among them, as shown in Fig. 4. The detailed results are presented in Table IV.

2) Generally, as we increase the tolerance, we increase the likelihood and number of quasi-optimal alternatives, but we lose relatively to the best position attained. Results show that it would take a very large value for the tolerance value to ensure a near-certainty probability of keeping the supposedly best alternative.
3) A tolerance of $\epsilon=0.10$ seems to be a good compromise, as there exists a reasonable probability $(17 \%-44 \%$ of the cases) of obtaining one or two quasi-optimal alternatives, which include the supposedly best one in more than $90 \%$ of the cases for the dimensions tested. With a tolerance of 0.05 , it is highly probable that there are no quasi-optimal alternatives, whereas, with a tolerance of 0.2 , there are more quasi-optimal alternatives, but they become worse in terms of the supposedly true ranking. 
TABLE V

Number of Dominated (Tolerance $\epsilon=0$ ) Or Quasi-Dominated $(\epsilon=0.05, \epsilon=0.1$, And $\epsilon=0.2$ ) Alternatives That May Be Excluded

\begin{tabular}{|c|c|c|c|c|c|c|c|c|c|c|c|}
\hline \multicolumn{12}{|c|}{$\epsilon=0.05$} \\
\hline $\mathrm{n}$ & $\mathrm{m}$ & Mean & $\%<\frac{3 m}{4}$ & $\%\left[\frac{3 m}{4}, m-1[\right.$ & $\% m-1$ & $\% m$ & Mean & $\%<\frac{3 m}{4}$ & $\%\left[\frac{3 m}{4}, m-1[\right.$ & $\% m-1$ & $\% m$ \\
\hline \multirow[t]{5}{*}{5} & 5 & 2.63 & 79.58 & 0.00 & 20.42 & 0.00 & 2.97 & 69.14 & 0.00 & 30.86 & 0.00 \\
\hline & 10 & 6.78 & 66.42 & 22.30 & 11.28 & 0.00 & 7.55 & 44.36 & 32.22 & 23.42 & 0.00 \\
\hline & 15 & 11.22 & 52.56 & 40.44 & 7.00 & 0.00 & 12.39 & 22.22 & 57.48 & 20.30 & 0.00 \\
\hline & 25 & 20.34 & 18.18 & 77.74 & 4.08 & 0.00 & 22.17 & 1.02 & 81.88 & 17.10 & 0.00 \\
\hline & 50 & 43.97 & 1.42 & 96.70 & 1.88 & 0.00 & 47.03 & 0.00 & 83.24 & 16.76 & 0.00 \\
\hline \multirow[t]{5}{*}{10} & 5 & 2.07 & 89.16 & 0.00 & 10.84 & 0.00 & 2.52 & 66.90 & 0.00 & 18.10 & 0.00 \\
\hline & 10 & 5.71 & 84.70 & 11.74 & 3.56 & 0.00 & 6.80 & 66.12 & 22.98 & 10.90 & 0.00 \\
\hline & 15 & 9.70 & 78.52 & 19.10 & 2.38 & 0.00 & 11.39 & 48.91 & 41.83 & 9.26 & 0.00 \\
\hline & 25 & 18.24 & 11.34 & 47.50 & 1.14 & 0.00 & 20.95 & 10.92 & 81.56 & 7.52 & 0.00 \\
\hline & 50 & 40.57 & 20.14 & 79.46 & 0.40 & 0.00 & 45.34 & 0.20 & 94.16 & 5.64 & 0.00 \\
\hline \multirow[t]{5}{*}{15} & 5 & 1.82 & 92.70 & 0.00 & 7.30 & 0.00 & 2.31 & 85.78 & 0.00 & 14.22 & 0.00 \\
\hline & 10 & 5.12 & 90.38 & 7.24 & 2.38 & 0.00 & 6.42 & 73.56 & 18.18 & 8.26 & 0.00 \\
\hline & 15 & 8.83 & 86.92 & 11.90 & 1.18 & 0.00 & 10.88 & 60.32 & 33.94 & 5.74 & 0.00 \\
\hline & 25 & 16.89 & 69.32 & 30.30 & 0.42 & 0.00 & 20.20 & 20.72 & 75.26 & 4.02 & 0.00 \\
\hline & 50 & 38.26 & 42.08 & 57.82 & 0.10 & 0.00 & 44.28 & 1.06 & 95.84 & 3.10 & 0.00 \\
\hline
\end{tabular}

$\epsilon=0.1 \quad \epsilon=0.2$

\begin{tabular}{|c|c|c|c|c|c|c|c|c|c|c|c|}
\hline $\mathrm{n}$ & $\mathrm{m}$ & Mean & $\%<\frac{3 m}{4}$ & $\%\left[\frac{3 m}{4}, m-1[\right.$ & $\% m-1$ & $\% m$ & Mean & $\%<\frac{3 m}{4}$ & $\%\left[\frac{3 m}{4}, m-1[\right.$ & $\% m-1$ & $\% m$ \\
\hline \multirow[t]{5}{*}{5} & 5 & 3.26 & 57.26 & 0.00 & 42.74 & 0.00 & 3.61 & 34.54 & 0.00 & 65.46 & 0.00 \\
\hline & 10 & 8.13 & 21.72 & 38.46 & 39.82 & 0.00 & 8.44 & 9.46 & 36.06 & 54.36 & 0.12 \\
\hline & 15 & 13.13 & 4.94 & 54.94 & 40.12 & 0.00 & 13.28 & 2.48 & 51.82 & 45.68 & 0.02 \\
\hline & 25 & 23.12 & 0.02 & 58.94 & 41.00 & 0.04 & 22.94 & 0.00 & 68.76 & 31.14 & 0.10 \\
\hline & 50 & 48.19 & 0.00 & 55.48 & 44.46 & 0.06 & 47.26 & 0.00 & 85.28 & 14.68 & 0.04 \\
\hline \multirow[t]{5}{*}{10} & 5 & 2.94 & 70.00 & 0.00 & 30.00 & 0.00 & 3.51 & 41.54 & 0.00 & 58.46 & 0.00 \\
\hline & 10 & 7.71 & 38.06 & 36.52 & 25.42 & 0.00 & 8.43 & 10.18 & 35.42 & 54.32 & 0.08 \\
\hline & 15 & 12.64 & 15.82 & 58.60 & 25.58 & 0.00 & 13.33 & 1.86 & 50.10 & 47.90 & 0.14 \\
\hline & 25 & 22.65 & 0.14 & 73.76 & 26.10 & 0.00 & 23.10 & 0.00 & 62.46 & 37.38 & 0.16 \\
\hline & 50 & 47.70 & 0.00 & 71.26 & 28.72 & 0.02 & 47.59 & 0.00 & 78.58 & 21.32 & 0.10 \\
\hline \multirow[t]{5}{*}{15} & 5 & 2.78 & 45.2 & 0.00 & 24.80 & 0.00 & 3.45 & 45.32 & 0.00 & 54.68 & 0.00 \\
\hline & 10 & 7.50 & 47.22 & 33.80 & 20.96 & 0.00 & 8.40 & 11.04 & 36.20 & 52.60 & 0.16 \\
\hline & 15 & 12.42 & 20.94 & 58.52 & 20.54 & 0.00 & 13.33 & 2.18 & 49.12 & 48.54 & 0.16 \\
\hline & 25 & 22.38 & 0.54 & 78.44 & 21.02 & 0.00 & 23.12 & 0.00 & 61.88 & 37.88 & 0.24 \\
\hline & 50 & 47.43 & 0.00 & 97.42 & 22.56 & 0.02 & 47.67 & 0.00 & 76.84 & 23.00 & 0.16 \\
\hline
\end{tabular}

TABLE VI

Best Position Attained by the Nonexcluded Alternatives (Tolerance $\epsilon=0.05, \epsilon=0.1$, And $\epsilon=0.2$ )

\begin{tabular}{|c|c|c|c|c|c|c|c|c|c|c|c|c|c|c|c|}
\hline \multicolumn{4}{|c|}{$\epsilon=0.05$} & \multicolumn{6}{|c|}{$\epsilon=0.1$} & \multicolumn{6}{|c|}{$\epsilon=0.2$} \\
\hline $\mathrm{n}$ & $\mathrm{m}$ & Mean & $\% 1$ & $\% 2$ & $\%>3$ & Mean & $\% 0$ & $\% 1$ & $\% 2$ & $\%>3$ & Mean & $\% 0$ & $\% 1$ & $\% 2$ & $\%>3$ \\
\hline 5 & 5 & 1.00 & 99.80 & 0.20 & $0 . \overline{00}$ & 1.01 & 0.00 & 98.80 & 1.16 & $0 . \overline{04}$ & 1.06 & 0.00 & 94.42 & 4.94 & $0 . \overline{64}$ \\
\hline & 10 & 1.01 & 99.98 & 0.72 & 0.02 & 1.05 & 0.00 & 95.62 & 3.98 & 0.40 & - & 0.08 & 89.28 & 8.48 & 2.16 \\
\hline & 15 & 1.01 & 98.86 & 1.10 & 0.04 & - & 0.06 & 94.06 & 4.88 & 1.00 & - & 0.16 & 87.90 & 9.02 & 2.92 \\
\hline & 25 & 1.02 & 98.42 & 1.56 & 0.02 & - & 0.02 & 91.20 & 7.28 & 1.50 & - & 0.08 & 87.10 & 8.70 & 4.12 \\
\hline & 50 & 1.04 & 96.80 & 2.88 & 0.32 & - & 0.06 & 87.88 & 8.90 & 3.16 & - & 0.02 & 88.44 & 7.78 & 3.76 \\
\hline 10 & 5 & 1.00 & 100.00 & 0.00 & 0.00 & 1.01 & 0.00 & 99.48 & 0.52 & 0.00 & 1.07 & 0.00 & 93.80 & 5.44 & 0.76 \\
\hline & 10 & 1.00 & 99.98 & 0.02 & 0.00 & 1.02 & 0.00 & 98.20 & 1.70 & 0.10 & - & 0.08 & 85.06 & 11.12 & 3.74 \\
\hline & 15 & 1.00 & 99.90 & 0.10 & 0.00 & 1.04 & 0.00 & 96.48 & 3.22 & 0.30 & - & 0.10 & 82.32 & 11.66 & 5.58 \\
\hline & 25 & 1.00 & 99.72 & 0.26 & 0.02 & 1.07 & 0.00 & 94.32 & 4.92 & 0.78 & - & 0.18 & 80.56 & 12.76 & 6.50 \\
\hline & 50 & 1.00 & 99.72 & 0.26 & 0.02 & - & 0.02 & 91.34 & 7.00 & 1.64 & - & 0.14 & 80.40 & 11.60 & 7.86 \\
\hline 15 & 5 & 1.00 & 100.00 & 0.00 & 0.00 & 1.00 & 0.00 & 99.58 & 0.42 & 0.00 & 1.11 & 0.00 & 90.90 & 7.74 & 1.36 \\
\hline & 10 & 1.00 & 99.98 & 0.02 & 0.00 & 1.02 & 0.00 & 98.08 & 1.86 & 0.06 & - & 0.16 & 81.54 & 12.84 & 5.46 \\
\hline & 15 & 1.00 & 99.90 & 0.10 & 0.00 & 1.04 & 0.00 & 96.44 & 3.10 & 0.46 & - & 0.14 & 78.60 & 13.44 & 7.82 \\
\hline & 25 & 1.00 & 99.90 & 0.10 & 0.00 & 1.07 & 0.00 & 94.20 & 4.82 & 0.98 & - & 0.26 & 74.76 & 14.34 & 10.64 \\
\hline & 50 & 1.00 & 99.88 & 0.12 & 0.00 & - & 0.04 & 97.76 & 6.98 & 2.22 & - & 0.18 & 72.44 & 14.60 & 12.78 \\
\hline
\end{tabular}

\section{Quasi-Dominance Rule}

The third set of experiments studied the use of the quasidominance relation for a given tolerance $\epsilon$ (recall Section II-B). The idea now is to retain the alternatives that are not quasidominated. As in the previous experiments, our purpose was to observe whether there exists a good value for $\epsilon$ such that it would allow retaining a few alternatives without discarding the supposedly best one. In these experiments, we sought to know how many alternatives would be retained, as well as the best position reached by the retained alternatives. Table $\mathrm{V}$ indicates the minimum, maximum, and mean number of dominated or quasi-dominated alternatives (to be excluded), as well as some particular quantiles, for different tolerance values. Conclusions are summarized here.

1) As the tolerance increases, so does the number of alternatives that may be excluded (including, in rare cases, the possibility of no alternative remaining, as stated in
Section II-B). On the other hand, Table VI indicates that the proportion of cases where we exclude the supposedly best alternative also increases (Fig. 5).

2) For a tolerance of 0.1 , the number of alternatives that can be excluded is high (more than three fourths of the total number) in almost all the cases; often, only one alternative remains $(21 \%-45 \%$ of the cases), whereas the proportion of cases where the best alternative is not quasidominated is always above $90 \%$ (with an exception).

3 ) As the number of alternatives increases, the probability of excluding more than three fourths of the alternatives rapidly increases, although the probability of keeping just one alternative also rapidly decreases.

Of interest in the last observation is that, under the rankorder constraint (3), dominance coincides with cumulative dominance [35]. According to the theoretical analysis of Baucells $e t$ al., by guaranteeing that an alternative $a$ is better 

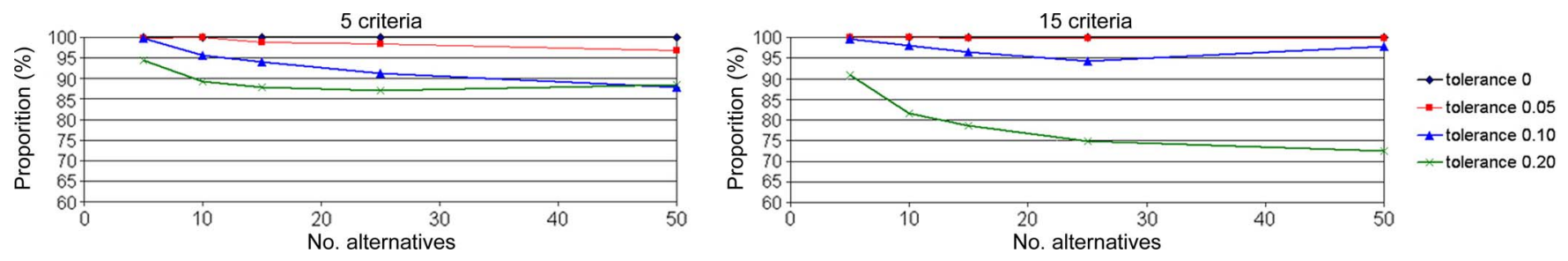

Fig. 5. Proportion of cases (in percent) where the best alternative is not quasi-dominated (cases with 5 and 15 criteria).

ranked than another alternative $b$ whenever $a$ cumulatively dominates $b$ (see [35] for details), the ROC rule should be particularly effective when cumulative dominance holds for many pairs of alternatives. As Table $\mathrm{V}$ indicates that the proportion of dominated alternatives $(\epsilon=0)$ rapidly increases with the number of alternatives, this helps in explaining the superiority of the ROC rule in the experiments presented in Section IV-A.

Comparing the strategies based on quasi-optimality and quasi-dominance for the same tolerance value $(\epsilon=0.10)$, the strategy for keeping the quasi-optimal alternatives has a lower probability of being usable (in many cases, there are no such alternatives), but, when it is usable, it selects a very small set (one or two alternatives most of the time) containing the supposedly best alternative in the vast majority of the cases. The strategy for keeping the nonquasi-dominated alternatives results in larger subsets, but the probability of not being usable is very low. A good combined strategy would then be to start by testing if there are quasi-optimal alternatives and, if there is none, to exclude the quasi-dominated ones.

\section{CONCLUSION}

We have performed a series of Monte Carlo simulations to study and compare the behavior of different decision rules for cases where there is only ordinal information about the weights of the additive MAUT/MAVT model. These experiments extend those found in the literature in two ways: 1) They tested strategies for selecting more than one alternative. 2) They tested decision rules based on the concepts of quasi-optimality and quasi-dominance.

For a strategy for selecting only one alternative, our results corroborate those found in the literature [15], [33], stating that, when we have a ranking of the weights, then the ROC weight rule is the best rule to be used, particularly as the number of criteria increases. Furthermore, this rule has the advantage of being easy to understand and implement. This superiority of the ROC rule would be expected when cumulative dominance [35] occurs for many pairs, which our results confirm to be the case, particularly as the number of alternatives increases. If the top two or three alternatives according to the ROC weights are chosen, the chances of selecting the supposedly best one significantly increases to at least $90 \%$ and $95 \%$, respectively.

The drawback of the ROC weight rule is that it does not convey any information about the potential loss of value associated with selecting one or more of the top-ranked alternatives with respect to the supposedly best one. To monitor this concern, the minimax regret, quasi-optimality, and quasi-dominance rules may be used. Our experiments indicate that the minimax regret rule is clearly inferior to the ROC weight rule, but the quasioptimality and quasi-dominance rules can yield results that are nearly as good as that of the latter. Thus, we suggested a strategy of starting by testing if there exist quasi-optimal alternatives and, if there is none, excluding quasi-dominated ones. We also suggested using a tolerance of 0.10 , which may be decreased if such a potential loss of value is considered excessive.

The conclusions from our experiments must be read with caution since they pertain to the well-known problem of deciding based on a ranking of the criteria's weights. For a case where the acceptable weights are defined by a general set of linear constraints, then it is possible that the ROC weights will lose some of their appeal, whereas the ideas of quasi-optimality and quasi-dominance may maintain results that are as good as the ones we have achieved. Investigating this conjecture seems to be an interesting future research.

Future research is also needed for cases where there also exists imprecise information on the performance value of each alternative under each criterion. Some experiments not included in this paper indicate that, if the information available is merely a ranking of the alternatives under each criterion, then the tested rules (adapted for these situations) naturally perform worse [36]. The ROC weight rule still yields interesting results, but, for instance, the hit ratio decreases from values in the range of $76 \%-88 \%$ to values in the range of $70 \%-78 \%$.

Another issue that we did not deal with is the final selection of one alternative from a subset of alternatives selected by a rule. In some cases, this might not be necessary if the purpose of the decision process is precisely to obtain a short list of candidates or if the quasi-optimality or quasi-dominance rules identify a single choice. In other cases, the process is continued, but, since there are a few alternatives now, they can more thoroughly be studied, reassessing their performances or even refining the set of criteria. As a general guideline, however, the process would continue by progressively eliciting more information, as confirmed in [16] and [23].

\section{ACKNOWLEDGMENT}

This research was motivated by a suggestion of W. Edwards when L.C. Dias presented the VIP Analysis Program at the Cincinnati INFORMS Spring 1999 Conference. The authors would like to thank the anonymous referees, Associated Editor, and Editor-in-Chief for their constructive comments and suggestions. 


\section{REFERENCES}

[1] V. Belton and T. J. Stewart, Multiple Criteria Decision Analysis: An Integrated Approach. Dordrecht, The Netherlands: Kluwer, 2002.

[2] Multiple Criteria Decision Analysis: State of the Art Surveys, J. Figueira, S. Greco, and M. Ehrgott, Eds. New York: Springer-Verlag, 2005.

[3] R. L. Keeney, Value-Focused Thinking. A Path to Creative Decision Making. Cambridge, MA: Harvard Univ. Press, 1992.

[4] R. L. Keeney and H. Raiffa, Decisions With Multiple Objectives: Preferences and Value Tradeoffs. New York: Wiley, 1976. (new edition by Cambridge University Press, Cambridge, 1993).

[5] D. von Winterfeldt and W. Edwards, Decision Analysis and Behavioral Research. Cambridge, MA: Cambridge Univ. Press, 1986.

[6] P. P. Wakker, Additive Representations of Preferences-A New Foundation of Decision Analysis. Dordrecht, The Netherlands: Kluwer, 1989.

[7] P. C. Fishburn, "Analysis of decisions with incomplete knowledge of probabilities," Oper. Res., vol. 13, no. 2, pp. 217-237, Mar./Apr. 1965.

[8] R. Clemen and L. Winkler, "Combining probability distributions from experts in risk analysis," Risk Anal., vol. 19, no. 2, pp. 187-203, Apr. 1999.

[9] K. Miettinen, Nonlinear Multiobjective Optimization. Boston, MA: Kluwer, 1999.

[10] M. Pöyhönen and R. P. Hämäläinen, "On the convergence of multiattribute weighting methods," Eur. J. Oper. Res., vol. 129, no. 3, pp. 569-585, Mar. 2001.

[11] P. J. H. Schoemaker and C. D. Waid, "An experimental comparison of different approaches to determining weights in additive utility models," Manage. Sci., vol. 28, no. 2, pp. 182-196, Feb. 1982.

[12] M. Weber and K. Borcherding, "Behavioural influences on weight judgments in multiattribute decision making," Eur. J. Oper. Res., vol. 67, no. 1, pp. 1-12, May 1993.

[13] W. Edwards and F. H. Barron, "SMARTS and SMARTER: Improved simple methods for multiattribute utility measurement," Organ. Behav. Human Decis. Process., vol. 60, no. 3, pp. 306-325, Dec. 1994.

[14] W. Edwards, D. von Winterfeldt, and D. L. Moody, "Simplicity in decision analysis: An example and an application," in Decision Making: Descriptive, Normative, and Prescriptive Approaches, D. E. Bell, H. Raiffa, and A. Tversy, Eds. Cambridge, MA: Cambridge Univ. Press, 1988, pp. 443-464.

[15] B. S. Ahn and K. S. Park, "Comparing methods for multiattribute decision making with ordinal weights," Comput. Oper. Res., vol. 35, no. 5, pp. 1660-1670, May 2008.

[16] L. C. Dias and J. N. Clímaco, "Additive aggregation with variable interdependent parameters: The VIP Analysis software," J. Oper. Res. Soc., vol. 51, no. 9, pp. 1070-1082, Sep. 2000.

[17] G. B. Hazen, "Partial information, dominance and potential optimality in multiattribute utility theory," Oper. Res., vol. 34, no. 2, pp. 297-310, Mar./Apr. 1986.

[18] A. A. Salo and R. P. Hämäläinen, "Preference ratios in multiattribute evaluation (PRIME)—Elicitation and decision procedures under incomplete information," IEEE Trans. Syst., Man, Cybern. A, Syst., Humans, vol. 31, no. 6, pp. 533-545, Nov. 2001.

[19] M. Weber, "Decision making with incomplete information," Eur. J. Oper. Res., vol. 28, no. 1, pp. 44-57, Jan. 1987.

[20] N. S. Iyer, "A family of dominance rules for multiattribute decision making under uncertainty," IEEE Trans. Syst., Man, Cybern. A, Syst., Humans, vol. 33, no. 4, pp. 441-450, Jul. 2003.

[21] K. S. Park, "Mathematical programming models for characterizing dominance and potential optimality when multicriteria alternative values and weights are simultaneously incomplete," IEEE Trans. Syst., Man, Cybern. A, Syst., Humans, vol. 34, no. 5, pp. 601-614, Sep. 2004.

[22] A. P. Sage and C. C. White, III, "ARIADNE: A knowledge-based interactive system for planning and decision support," IEEE Trans. Syst., Man, Cybern. A, Syst., Humans, vol. SMCA-14, no. 1, pp. 35-47, Jan./Feb. 1984.

[23] C. C. White, Jr. and H. A. Holloway, "Resolvability for imprecise multiattribute alternative selection," IEEE Trans. Syst., Man, Cybern. A, Syst., Humans, vol. 38, no. 1, pp. 162-169, Jan. 2008.

[24] M. Baucells and R. K. Sarin, "Group decisions with multiple criteria," Manage. Sci., vol. 49, no. 8, pp. 1105-1118, Aug. 2003.

[25] L. C. Dias and J. N. Clímaco, "Dealing with imprecise information in group multicriteria decisions: A methodology and a GDSS architecture," Eur. J. Oper. Res., vol. 160, no. 2, pp. 291-307, Jan. 2005.
[26] S. H. Kim and B. S. Ahn, "Interactive group decision making procedure under incomplete information," Eur. J. Oper. Res., vol. 116, no. 3, pp. 498-508, Aug. 1999.

[27] A. A. Salo, "Interactive decision aiding for group decision support," Eur. J. Oper. Res., vol. 84, no. 1, pp. 134-149, Jul. 1995.

[28] Y. Chen, K. W. Hipel, and D. M. Kilgour, "Multiple criteria sorting using case-based distance models with an application in water resources management," IEEE Trans. Syst., Man, Cybern. A, Syst., Humans, vol. 37 , no. 5, pp. 4680-4691, Sep. 2007.

[29] L. C. Dias and V. Mousseau, "IRIS: A DSS for multiple criteria sorting problems," J. Multi-Criteria Decis. Anal., vol. 12, pp. 285-298, 2003.

[30] B. Malakooti, "Ranking and screening multiple criteria alternatives with partial information and use of ordinal and cardinal strength of preferences," IEEE Trans. Syst., Man, Cybern. A, Syst., Humans, vol. 30, no. 3, pp. 355-368, May 2000.

[31] B. S. Ahn, "Extending Malakooti's model for ranking multicriteria alternatives with preference strength and partial information," IEEE Trans. Syst., Man, Cybern. A, Syst., Humans, vol. 33, no. 3, pp. 281-287, May 2003.

[32] B. S. Ahn, "Multi-attribute decision aid with extended ISMAUT," IEEE Trans. Syst., Man, Cybern. A, Syst., Humans, vol. 36, no. 3, pp. 507-520, May 2006.

[33] F. H. Barron and B. E. Barrett, "Decision quality using ranked attribute weights," Manage. Sci., vol. 42, no. 11, pp. 1515-1523, Nov. 1996.

[34] A. Salo and A. Punkka, "Decision aiding-Rank inclusion in criteria hierarchies," Eur. J. Oper. Res., vol. 163, no. 2, pp. 338-356, Jun. 2005.

[35] M. Baucells, J. A. Carrasco, and R. M. Hogarth, "Cumulative dominance and heuristic performance in binary multiattribute choice," Oper. Res., vol. 56, no. 5, pp. 1289-1304, Sep./Oct. 2008.

[36] P. Sarabando Santos, "Comparação de métodos multicritério de avaliação com informação ordinal utilizando simulação Monte Carlo," Dissertação de Mestrado, Faculdade de Economia da Univ. Coimbra, Coimbra, Portugal, 2006. (in Portuguese).

[37] J. Butler, J. Jia, and J. Dyer, "Simulation techniques for the sensitivity analysis of multi criteria decision models," Eur. J. Oper. Res., vol. 103, no. 3, pp. 531-546, Dec. 1997.

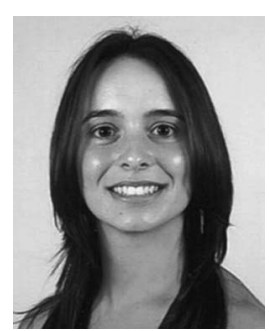

Paula Sarabando received the B.S. degree in mathematics and the M.Sc. degree in information management from the University of Coimbra, Coimbra, Portugal, in 2003 and 2006, respectively. She is currently working toward the Ph.D. degree at the University of Coimbra.

She is currently a Lecturer with the Escola Superior de Tecnologia de Viseu, Viseu, Portugal.

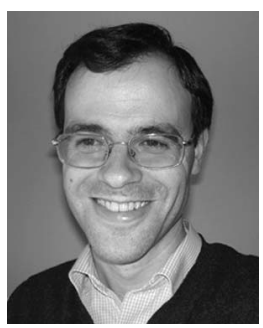

Luís Cândido Dias received the Licentiate degree in informatics engineering and the Ph.D. degree in management/systems sciences from the University of Coimbra, Coimbra, Portugal, in 1992 and 2001, respectively. He is currently with the Faculty of Economics, University of Coimbra, where he teaches decision analysis, operations research, and informatics. He is also a Researcher of the INESC Coimbra R\&D Institute, where he was part of the Board of Directors from 2001 to 2007. He has published numerous papers in journals such as Computers and Operations Research, Decision Support Systems, Energy Policy, European Journal of Operational Research, Group Decision and Negotiation, Journal of Global Optimization, Journal of Multi-Criteria Decision Analysis, or Journal of the Operational Research Society. His research interests include multicriteria decision analysis, group decision and negotiation support, and decision support systems. 